\title{
Feasibility of Sentinel Node Biopsy in Head and Neck Melanoma Using a Hybrid Radioactive and Fluorescent Tracer
}

\author{
Oscar R. Brouwer, $\mathrm{MD}^{1}$, W. Martin C. Klop, $\mathrm{MD}^{2}$, Tessa Buckle, $\mathrm{MSc}^{3}$, Lenka Vermeeren, $\mathrm{MD}^{1}$, \\ Michiel W. M. van den Brekel, $\mathrm{MD}^{2}$, Alfons J. M. Balm, $\mathrm{MD}^{2}$, Omgo E. Nieweg, $\mathrm{MD}^{4}$, \\ Renato A. Valdés Olmos, $\mathrm{MD}^{1}$, and Fijs W. B. van Leeuwen, $\mathbf{P h D}^{3}$
}

${ }^{1}$ Department of Nuclear Medicine, The Netherlands Cancer Institute, Amsterdam, The Netherlands; ${ }^{2}$ Department of Head \& Neck Surgery and Oncology, The Netherlands Cancer Institute, Amsterdam, The Netherlands; ${ }^{3}$ Department of Radiology, Leiden University Medical Center, Leiden, The Netherlands; ${ }^{4}$ Department of Surgery, The Netherlands Cancer Institute, Amsterdam, The Netherlands

\begin{abstract}
Purpose. This study was designed to examine the feasibility of combining lymphoscintigraphy and intraoperative sentinel node identification in patients with head and neck melanoma by using a hybrid protein colloid that is both radioactive and fluorescent.

Methods. Eleven patients scheduled for sentinel node biopsy in the head and neck region were studied. Approximately $5 \mathrm{~h}$ before surgery, the hybrid nanocolloid labeled with indocyanine green (ICG) and technetium-99m $\left({ }^{99 \mathrm{~m}} \mathrm{Tc}\right)$ was injected intradermally in four deposits around the scar of the primary melanoma excision. Subsequent lymphoscintigraphy and single photon emission computed tomography with computed tomography (SPECT/CT) were performed to identify the sentinel nodes preoperatively. In the operating room, patent blue dye was injected in 7 of the 11 patients. Intraoperatively, sentinel nodes were acoustically localized with a gamma ray detection probe and visualized by using patent blue dye and/or fluorescencebased tracing with a dedicated near-infrared light camera. A portable gamma camera was used before and after sentinel node excision to confirm excision of all sentinel nodes.
\end{abstract}

Electronic supplementary material The online version of this article (doi:10.1245/s10434-011-2180-7) contains supplementary material, which is available to authorized users.

(C) The Author(s) 2011. This article is published with open access at Springerlink.com

First Received: 2 August 2011;

Published Online: 30 December 2011

F. W. B. van Leeuwen, $\mathrm{PhD}$

e-mail: F.W.B.van_Leeuwen@lumc.nl
Results. A total of 27 sentinel nodes were preoperatively identified on the lymphoscintigraphy and SPECT/CT images. All sentinel nodes could be localized intraoperatively. In the seven patients in whom blue dye was used, $43 \%$ of the sentinel nodes stained blue, whereas all were fluorescent. The portable gamma camera identified additional sentinel nodes in two patients. Ex vivo, all radioactive lymph nodes were fluorescent and vice versa, indicating the stability of the hybrid tracer.

Conclusions. ICG- ${ }^{99 \mathrm{~m}} \mathrm{Tc}$-nanocolloid allows for preoperative sentinel node visualization and concomitant intraoperative radio- and fluorescence guidance to the same sentinel nodes in head and neck melanoma patients.

The intricate anatomy and the presence of several vital structures in the neck render lymphatic mapping in this region more difficult than elsewhere. ${ }^{1,2}$ The limited value of vital dye compared with other regions means that the surgeon has to rely mainly on the preoperative lymphoscintigraphy and the intraoperative gamma ray detection probe. ${ }^{3-5}$ As a result, the sentinel node identification rate is relatively low and the false-negative rate is high. ${ }^{6}$ Single photon emission computerized tomography with computed tomography (SPECT/CT) provides three-dimensional images and shows the sentinel node in its anatomic habitat, facilitating the preoperative planning of the surgical approach. ${ }^{7,8}$ An additional intraoperative detection tool could improve the identification rate considerably, because sentinel nodes in the head and neck region often are located in close proximity to the injection site and high background signals can hamper sentinel node detection with the probe. A portable gamma camera was introduced with a better spatial resolution than the probe, and initial studies showed promising results in the 
intraoperative visualization of radioactive sentinel nodes, but this device cannot depict the surrounding anatomy., ${ }^{9,10}$

Some surgeons, including us, prefer not to use vital blue dyes in the face to prevent long-term tattooing effects. ${ }^{11}$ The fact that near-infrared fluorescent dyes, such as indocyanine green (ICG), are invisible to the human eye avoids this drawback. ${ }^{12}$ Moreover, near-infrared dyes have an improved detection sensitivity and tissue penetration, which may facilitate the detection of a sentinel node and its afferent lymphatic duct. ${ }^{13-16}$ Yet like vital blue dye, ICG migrates quickly through the lymphatic system, resulting in a limited diagnostic time window. ${ }^{17}$

To address the limitations of current radioguided and vital dye-based intraoperative sentinel node detection, we developed a hybrid tracer by combining ICG and ${ }^{99 m} \mathrm{Tc}$ nanocolloid into ICG- ${ }^{99} \mathrm{~m}$ Tc-nanocolloid. This tracer is both radioactive and fluorescent and exhibits the same macrophage-based accumulation in lymph nodes as the ${ }^{99 \mathrm{~m}} \mathrm{Tc}$ labeled radiocolloid. ${ }^{18}$ It permits sentinel node detection using conventional lymphoscintigraphy and SPECT/CT, as well as fluorescence imaging, which was initially shown in laparoscopic sentinel node biopsy in prostate cancer patients. ${ }^{19}$ The current study of patients with a melanoma in the head and neck region is the first to explore this new approach in open sentinel node procedures. The main purpose of the study was to examine whether the hybrid tracer enables successful lymphoscintigraphy, SPECT/CT, and intraoperative radio- and fluorescence guidance to all the sentinel nodes that were visualized preoperatively. A comparison to patent blue was made in patients in whom both tracers were used.

\section{METHODS}

\section{Patients}

Patient characteristics are outlined in Table 1. Eleven patients were prospectively enrolled after a diagnostic excision with 2-mm margins of a melanoma in the head or neck with a Breslow thickness of at least $1 \mathrm{~mm}$ or Clark level IV. All patients were clinically node-negative (palpation, ultrasound, cytology) and were scheduled for sentinel node biopsy and reexcision of the melanoma scar. The mean age of the patients was 54 (range 32-75) years. The study protocol was approved by the Institutional Review Board, and all patients were included after giving informed consent.

\section{Tracer Preparation}

After the preparation of ${ }^{99 \mathrm{~m}} \mathrm{Tc}$-nanocolloid (GE Healthcare, Eindhoven, The Netherlands), hybrid ICG- ${ }^{99 \mathrm{~m}} \mathrm{Tc}$ nanocolloid was formed using a dose of $0.25 \mathrm{mg}$ of ICG (ICG-Pulsion, Pulsion Medical Systems, Munich, Germany) as previously described, with the exception of the addition of 2 -ml volume of saline during the ${ }^{99 \mathrm{~m}}$ Tc-nanocolloid preparation. ${ }^{19}$ This adaptation was made to achieve a nanocolloid concentration identical to the ${ }^{99 \mathrm{~m}}$ Tc-nanocolloid commonly used at our institution. All procedures were performed under good manufacturing practice (GMP-z) and under supervision of the institution's pharmacist.

\section{Tracer Administration and Preoperative Imaging}

A schematic overview of the study setup is depicted in Fig. 1. Four to $6 \mathrm{~h}$ before the operation, $70 \mathrm{MBq}$ ICG- ${ }^{99 m}$ Tc-nanocolloid in $0.4 \mathrm{ml}$ was injected intradermally in four deposits around the scar of the primary melanoma excision. Anterior and lateral dynamic images were obtained during the first $10 \mathrm{~min}$ after injection using a dual-head gamma camera (Symbia T, Siemens, Erlangen, Germany) to visualize the lymphatic duct. Static planar gamma camera images were acquired at $15 \mathrm{~min}$ and $2 \mathrm{~h}$ postinjection (Fig. 2a). SPECT/CT (Symbia T) was performed $2 \mathrm{~h}$ postinjection (Fig. 2b). After SPECT/CT fusion, the exact location of the sentinel node(s) was determined in relation to the surrounding anatomical structures. A sentinel node was defined as a lymph node on a direct lymphatic drainage pathway from the primary tumor. $^{20}$ The location of a sentinel node was marked on the skin to provide an external focal spot for the laser pointer of the portable gamma camera during the operation.

\section{Surgical Procedure}

Sentinel node biopsy and reexcision of the melanoma scar started within $6 \mathrm{~h}$ after injection of the tracer. Immediately before the operation, a mean volume of $1.0 \mathrm{ml}$ of patent blue dye (Laboratoire Guerbet, Aulnay-Sous-Bois, France) was injected intradermally at the primary tumor site in the seven patients with melanoma located outside the facial area. Subsequently, the sentinel nodes were visualized using a portable gamma camera (Sentinella, Oncovision, Valencia, Spain) to decide on the location for the incision(s) (Fig. 2c, d). Image acquisition times ranged from $30 \mathrm{~s}$ to $1 \mathrm{~min}$ in accordance with previous reports. ${ }^{10}$ After incision, the sentinel nodes were initially pursued with a hand-held gamma ray detection probe (Neoprobe, Johnson \& Johnson Medical, Hamburg, Germany). During surgical exploration, sentinel nodes were visualized using either the near-infrared fluorescence camera system (Photodynamic eye, Hamamatsu Photonics, Hamamatsu, Japan; Fig. 2e) or the blue dye. Fluorescence imaging required the lights in the operating room to be dimmed to minimize the background signal, whereas reflection of the excitation light was used to visualize the surrounding tissues (Fig. 2f). There are multiple near-infrared fluorescence cameras available. ${ }^{21}$ The cost- 
TABLE 1 Patient characteristics, sentinel node locations, and intraoperative findings

\begin{tabular}{|c|c|c|c|c|c|c|c|c|c|c|c|c|c|}
\hline Patient & $\begin{array}{l}\text { Age } \\
\text { (years) }\end{array}$ & Sex & $\begin{array}{l}\text { Melanoma } \\
\text { location }\end{array}$ & $\begin{array}{l}\text { Breslow } \\
(\mathrm{mm})\end{array}$ & $\begin{array}{l}\text { Clark } \\
\text { (level) }\end{array}$ & $\begin{array}{l}\text { No. of } \\
\text { SNs on } \\
\text { SPECT/ } \\
\text { CT }\end{array}$ & $\begin{array}{l}\text { Location of } \\
\text { SNs (on } \\
\text { SPECT/CT) }\end{array}$ & $\begin{array}{l}\text { SNs } \\
\text { localized } \\
\text { with } \\
\text { probe }\end{array}$ & $\begin{array}{l}\text { SNs } \\
\text { visualized } \\
\text { with } \\
\text { gamma } \\
\text { camera }\end{array}$ & $\begin{array}{l}\text { Blue } \\
\mathrm{SNs}^{\mathrm{a}}\end{array}$ & $\begin{array}{l}\text { Fluorescent } \\
\text { SNs }\end{array}$ & $\begin{array}{l}\text { Excised } \\
\text { SNs }\end{array}$ & $R^{2}$ \\
\hline 1 & 32 & Female & $\begin{array}{l}\text { Infraorbital } \\
\text { region (left) }\end{array}$ & 1.6 & IV & 1 & Level II left & 1 & 1 & $\mathrm{a}$ & 1 & 1 & $-{ }^{\mathrm{b}}$ \\
\hline 2 & 39 & Male & $\begin{array}{l}\text { Buccal } \\
\text { region (left) }\end{array}$ & 2.45 & III & 2 & $\begin{array}{l}\text { Level I left, level } \\
\text { II left }\end{array}$ & 2 & 2 & a & 2 & 2 & 0.79 \\
\hline 3 & 70 & Female & $\begin{array}{l}\text { Infraorbital } \\
\text { region } \\
\text { (right) }\end{array}$ & 1.2 & IV & 3 & $\begin{array}{l}\text { Level I right, } \\
\text { level II right } \\
\text { Level III left }\end{array}$ & 3 & 3 & $\mathrm{a}$ & 3 & 3 & 0.96 \\
\hline 4 & 66 & Female & $\begin{array}{l}\text { Infraorbital } \\
\text { region (left) }\end{array}$ & 0.9 & IV & 2 & $\begin{array}{l}\text { Level I left, level } \\
\text { II left }\end{array}$ & 2 & 2 & a & 0 & 2 & $-^{\mathrm{c}}$ \\
\hline 5 & 75 & Male & $\begin{array}{l}\text { Parietal } \\
\text { scalp } \\
\text { (medial) }\end{array}$ & 2.2 & $\mathrm{~V}$ & 3 & $\begin{array}{l}\text { Suboccipital } \\
\text { region left, level } \\
\text { II left } \\
\text { Level III right }\end{array}$ & 3 & 3 & 0 & 3 & 3 & 0.98 \\
\hline 6 & 50 & Male & $\begin{array}{l}\text { Neck } \\
\text { (posterior } \\
\text { left) }\end{array}$ & 1.2 & IV & 1 & Level V left & 1 & 2 & 2 & 2 & 2 & 0.96 \\
\hline 7 & 46 & Male & $\begin{array}{l}\text { Parietal } \\
\text { scalp (left) }\end{array}$ & 0.9 & IV & 2 & $\begin{array}{l}\text { Occipital region } \\
\text { left, level V left }\end{array}$ & 2 & 2 & 2 & 2 & 2 & $-{ }^{\mathrm{d}}$ \\
\hline 8 & 32 & Female & $\begin{array}{l}\text { Parietal } \\
\text { scalp (left) }\end{array}$ & 4 & $\mathrm{~V}$ & 3 & $\begin{array}{l}\text { Level II left } \\
(\times 2), \text { level V left }\end{array}$ & 3 & 3 & 0 & 3 & 3 & 0.99 \\
\hline 9 & 56 & Female & $\begin{array}{l}\text { Parietal } \\
\text { scalp } \\
\text { (medial) }\end{array}$ & 5 & $\mathrm{~V}$ & 6 & $\begin{array}{l}\text { Level I left, level } \\
\text { II left, level V } \\
\text { left } \\
\text { Level I right, } \\
\text { level II right, } \\
\text { level V right }\end{array}$ & 6 & 6 & 1 & 6 & 6 & 0.77 \\
\hline 10 & 68 & Female & $\begin{array}{l}\text { Temporal } \\
\text { scalp (left) }\end{array}$ & 7 & IV & 2 & $\begin{array}{l}\text { Parotid region } \\
\text { left, level II left }\end{array}$ & $\begin{array}{l}1 \text { (level } \\
\text { II) }\end{array}$ & $\begin{array}{l}2 \text { (level } \\
\text { II) }\end{array}$ & 2 & 3 & 3 & 0.9 \\
\hline 11 & 56 & Male & $\begin{array}{l}\text { Temporal } \\
\text { scalp (left) }\end{array}$ & 2 & IV & 2 & $\begin{array}{l}\text { Parotid region } \\
\text { left, level II left }\end{array}$ & $\begin{array}{l}1 \text { (level } \\
\text { II) }\end{array}$ & $\begin{array}{l}1 \text { (level } \\
\text { II) }\end{array}$ & 2 & 2 & 2 & 0.96 \\
\hline
\end{tabular}

$S N$ sentinel node, $L N$ lymph node, $R^{2}$ correlation coefficient (squared) of ex vivo radioactive/fluorescent content in the excised lymph nodes

${ }^{a}$ Injection of patent blue was omitted in the four patients with a melanoma in the face

${ }^{\mathrm{b}}$ In this patient, only one lymph node was excised, so no correlation could be calculated

${ }^{c}$ In this patient, both the radioactive/fluorescent signal intensities were too weak for reliable $R^{2}$ calculation

${ }^{\mathrm{d}}$ In this patient, no $R^{2}$ was calculated

friendly camera used in the current study required no additional setup time other than the time needed for sterile draping and visualizes the fluorescent signal in real-time. After excision of a sentinel node, the portable gamma camera was used to search for potential additional sentinel nodes as previously described. ${ }^{10}$ Additional sentinel nodes were removed and second-echelon nodes were left in situ.

\section{Ex Vivo Specimen Analysis}

Quantitative ex vivo images of the excised tissue specimens were acquired with the portable gamma camera and with a more sensitive preclinical fluorescence camera system (IVIS200, Xenogen, Caliper Life Sciences, Hopkinton, MA) to determine the radioactive and fluorescent signal intensities as described previously. ${ }^{17-19}$ Trend line-based linear regression correlations were used to study the correlation between the radioactive and fluorescent signal intensities. All sentinel nodes were fixed in formalin, bisected, embedded in paraffin, and cut at a minimum of six levels at $50-$ to $150-\mu \mathrm{m}$ intervals. Pathologic evaluation included hematoxylin and eosin and immunohistochemical staining using S-100 and HMB-45. 
FIG. 1 Schematic overview of the study set-up. a After injection of ICG- ${ }^{99 \mathrm{~m}} \mathrm{Tc}$ nanocolloid, preoperative imaging of the sentinel nodes is performed. Intraoperatively, sentinel node localization is guided by the gamma ray detection probe, the portable gamma camera and the nearinfrared (NIR) fluorescence camera. Postexcision gamma camera imaging confirms complete excision of all sentinel nodes. $\mathbf{b}$ In the patients with melanoma outside the facial region, patent blue was intraoperatively injected for optical detection of blue-stained sentinel nodes and a comparison with fluorescence imaging was made

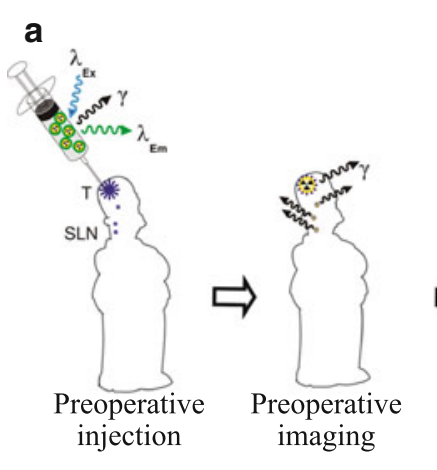

b
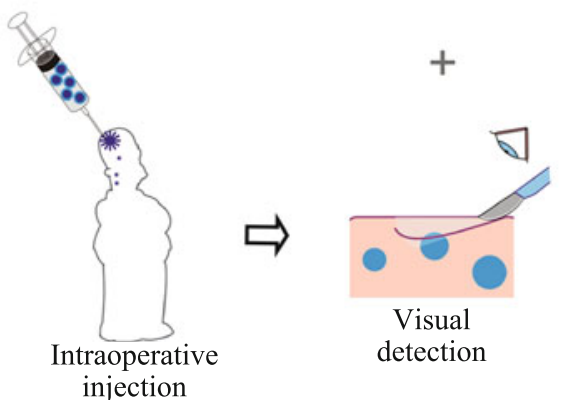
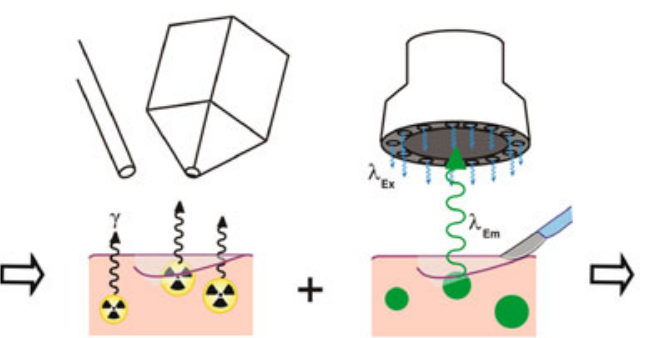

Gamma tracing/imaging

NIR fluorescence imaging
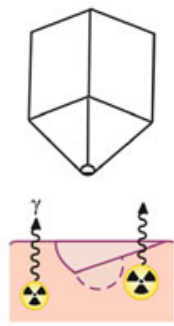

Post-excision gamma imaging

\section{RESULTS}

Characteristics and intraoperative findings of all 11 patients are outlined in Table 1. Preoperatively, conventional lymphoscintigraphy and SPECT/CT visualized a total of 27 sentinel nodes, at least 1 in each patient, with a mean of 2.5 per patient and a maximum of 6 . SPECT/CT provided accurate anatomical reference points to plan the surgical procedure in all patients.

Intraoperatively, the combination of radioactivity and fluorescence enabled localization and excision of all 27 preoperatively identified sentinel nodes. In patients 10 and 11 , a sentinel node in the parotid gland could neither be identified with the probe nor with the portable gamma camera due to the overwhelming background signal from the nearby injection site (temporal scalp, Table 1). These sentinel nodes were localized using the fluorescence camera. Fluorescence imaging visualized the sentinel nodes in 10 of 11 patients. Two sentinel nodes in patient 4 could not be visualized using the fluorescence camera, these nodes could still be localized using the portable gamma camera and probe despite the low radioactivity count rate compared with the background. Nevertheless, the more sensitive IVIS200 fluorescence camera did demonstrate a faint fluorescent signal in both nodes ex vivo. Two cases exemplifying the penetration of the fluorescent signal through the skin but also the substantial signal attenuation caused by a blood vessel are provided in the Supplementary information (Figs. S1-S2).

In the seven patients in whom blue dye was used, only 9 of the 21 sentinel nodes (43\%) stained blue, while all were fluorescent (Table 1). In two patients, the portable gamma camera revealed substantial residual radioactivity at the very location from which the sentinel node had been excised. Further exploration resulted in localization and removal of an additional sentinel node that was blue and fluorescent in patient 6 , and only fluorescent in patient 10 . Preoperative lymphoscintigraphy and SPECT/CT had not shown these sentinel nodes separately due to their close proximity. In hindsight, the corresponding CT images revealed two adjacent nodes at these locations (see Supplementary information Fig. S3).

Ex vivo, all radioactive lymph nodes were found to be fluorescent and vice versa, showing a strong intensity correlation with an average $R^{2}$ of 0.91 (Table 1). This indicates that the tracer is stable throughout the entire process. None of the excised lymph nodes were found to contain metastasis at histopathological examination.

\section{DISCUSSION}

This pilot study demonstrates the feasibility of combining preoperative sentinel node visualization with intraoperative radio- and fluorescence-guided sentinel node identification using a single injection of the hybrid tracer ICG- ${ }^{99 m} \mathrm{Tc}$ nanocolloid in open surgery, after this approach had been introduced in laparoscopic procedures. ${ }^{19}$ The preoperatively observed drainage patterns and the numbers of removed sentinel nodes were similar to the results in studies in which ${ }^{99 \mathrm{~m}} \mathrm{Tc}$-nanocolloid alone was used for lymphatic mapping, suggesting that that hybrid tracer behaves like the original radiocolloid. ${ }^{8}$ Intraoperatively, ICG- ${ }^{99 \mathrm{~m}}$ Tc-nanocolloid 

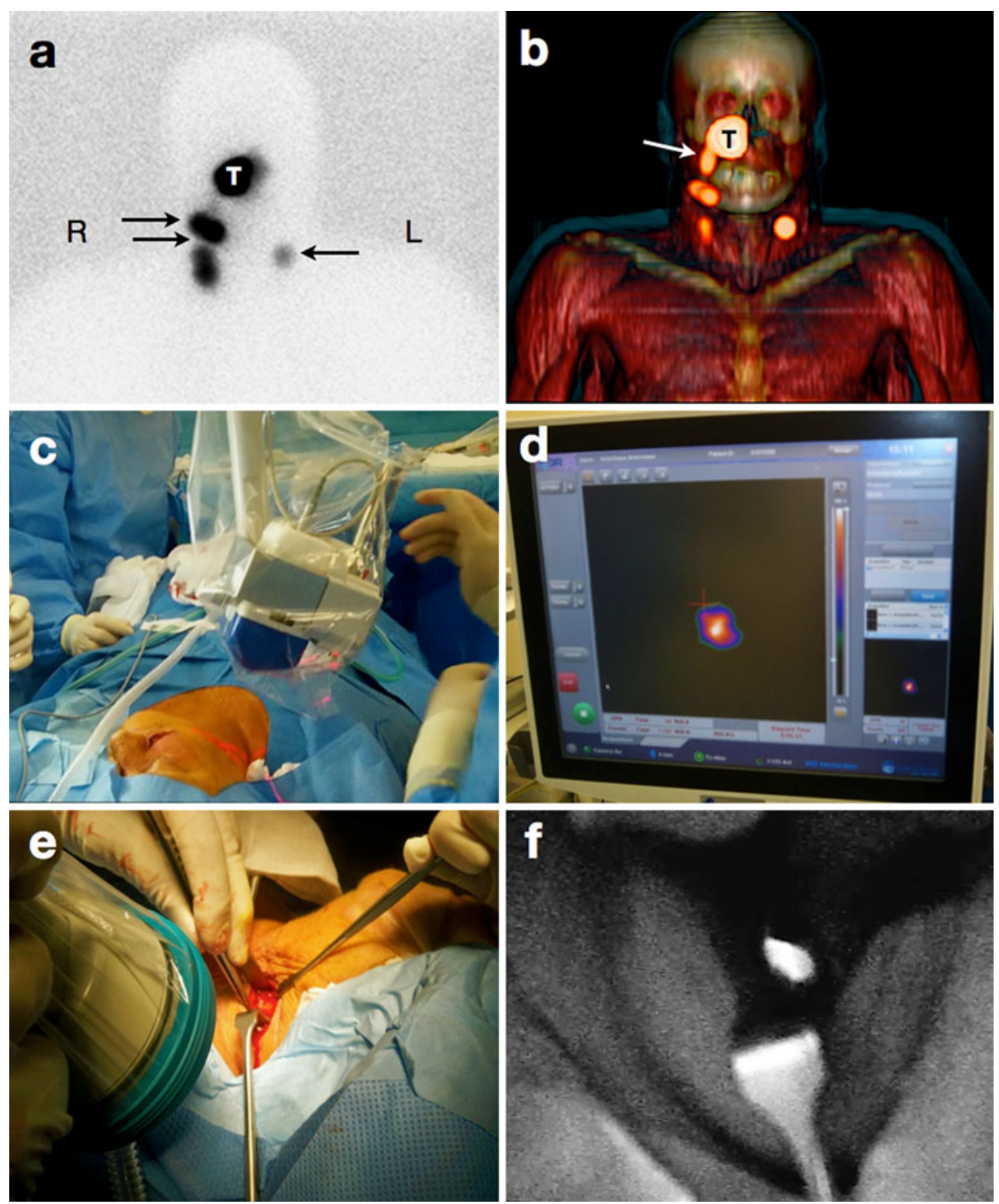

FIG. 2 Combined preoperative lymphatic mapping and intraoperative radio- and fluorescence-guided sentinel node biopsy (patient 3, Table 1). a Early static anterior preoperative lymphoscintigram at $10 \mathrm{~min}$ after infraorbital peritumoral injection of ICG- ${ }^{99 \mathrm{~m}} \mathrm{Tc}-$ nanocolloid showing the injection site $(\mathrm{T})$ with lymphatic drainage to two sentinel nodes in the neck on the right (R) side and a third one on the left (L) side (arrows). b 3D SPECT/CT image $2 \mathrm{~h}$ postinjection

enabled both radioguided and optical (fluorescence) guidance to the very sentinel nodes depicted on the preoperative images. Conventional techniques using optical dye(s) separately have several disadvantages. First, small organic dyes, such as vital blue dye or ICG alone quickly pass through the sentinel nodes downstream to subsequent nodes, which may be confusing and lead to the unnecessary removal of highertier nodes. ${ }^{17}$ The fast migration also may leave the sentinel node unstained after a while. ${ }^{4,5}$ The hybrid approach using

providing additional anatomical information with visualization of a lymphatic duct (arrow) originating from the injection site (T). c, $\mathbf{d}$ Intraoperatively, the radioactive component of the hybrid tracer in the left sentinel node is visualized using a portable gamma camera, and its laser pointer guides placement of the incision. e, $\mathbf{f} \mathrm{A}$ nearinfrared fluorescence camera is used to visualize the fluorescent component of the hybrid tracer in the same (non-blue) sentinel node

ICG- ${ }^{99 \mathrm{~m}} \mathrm{Tc}$-nanocolloid overcomes these drawbacks. The finding that the radioactive and fluorescent signal intensities overlap ex vivo (average $R^{2} 0.91$; Table 1) illustrates that the hybrid tracer remains stable throughout the entire process and largely remains trapped in the sentinel node. This results in a long intraoperative detection window of $6 \mathrm{~h}$ in the current study, and we have already seen in a separate study that the hybrid tracer is still effective after $20 \mathrm{~h}$ (unpublished data). In the head and neck region, lymph nodes are relatively 
small (3-4 mm), frequently clustered together, and often located close to the injection site. This renders sentinel node localization with the probe notoriously difficult. Blue or fluorescent dyes are not hampered by the background signal coming from the injection site and enable visual identification of the sentinel node, because it stands out among a cluster of lymph nodes and the surrounding structures. The current study confirms the findings of other investigators that only a limited percentage of the sentinel nodes stain blue in this region. ${ }^{2,4}$ In the present study, the $57 \%$ of nodes that did not stain blue were all intraoperatively visualized exploiting the fluorescent signature of the hybrid tracer.

The radioactive component of the hybrid tracer enables preoperative lymphoscintigraphy, which remains indispensable to identify the sentinel node in this area of unpredictable lymphatic drainage. ${ }^{22}$ Furthermore, the sequential early images are essential to identify a sentinel (first-echelon) node because some of the tracer may pass through to lodge in higher-echelon nodes. ${ }^{20}$ SPECT/CT is of particular value for surgical planning, because it visualizes the complex anatomy in the neck. ${ }^{8}$ Intraoperatively, the sensitive portable gamma camera enables the surgeon to obtain an image depicting all radioactive hot spots, which can serve as an additional reference irrespective of tissue movement or changes in patient position. ${ }^{9}$ After removal of the sentinel node(s), the portable gamma camera also can help assess whether all sentinel nodes have been removed, regardless of their location and depth. ${ }^{10}$ Illustrating this point, postexcision gamma ray imaging led to the identification of additional sentinel nodes in two patients in this series, although we cannot exclude that these nodes would have been found with the probe as well. These advantages of gamma ray imaging cannot be replaced by fluorescence imaging, because the near-infrared light can only penetrate a tissue layer of $1.5 \mathrm{~cm}$ at most. ${ }^{23}$

Taken together, radioactivity and fluorescence have complementary properties and their combination in the hybrid tracer ICG- ${ }^{99 \mathrm{~m}} \mathrm{Tc}$-nanocolloid facilitates sentinel node procedures in complex areas. This approach may be particularly valuable when sentinel nodes are located close to the injection site and in regions where small organic dyes, such as patent blue, are of limited value.

\section{CONCLUSIONS}

A single injection of the hybrid tracer ICG- ${ }^{99 \mathrm{~m}} \mathrm{Tc}$-nanocolloid enables preoperative sentinel node visualization and concomitant intraoperative radio- and fluorescence-guidance to the same sentinel nodes in head and neck melanoma patients. The fluorescent component in this hybrid approach offers a more generous time window than the currently used blue dye; it can be visualized through a layer of tissue and allows for optical detection of sentinel nodes that do not stain blue. A larger study is in progress to substantiate these preliminary findings.

ACKNOWLEDGMENT This research is supported, in part, by a KWF-Translational Research Award (Grant No. PGF 2009-4344; FvL) and via a FP7-HYPERImage (Grant No. 201651; TB). The authors gratefully acknowledge the entire surgical staff and the technicians of the nuclear medicine department for their contribution, and Professor T. J. M. Ruers for kindly providing the fluorescence camera system.

CONFLICT OF INTEREST The authors declare that they have no conflict of interest.

OPEN ACCESS This article is distributed under the terms of the Creative Commons Attribution Noncommercial License which permits any noncommercial use, distribution, and reproduction in any medium, provided the original author(s) and source are credited.

\section{REFERENCES}

1. Tanis PJ, Nieweg OE, van den Brekel MWM, Balm AJM. Dilemma of clinically node-negative head and neck melanoma: outcome of "watch and wait" policy, elective lymph node dissection, and sentinel node biopsy-a systematic review. Head Neck. 2008;30:380-9.

2. Jansen L, Koops HS, Nieweg OE, et al. Sentinel node biopsy for melanoma in the head and neck region. Head Neck. 2000;22:2733.

3. Kapteijn BA, Nieweg OE, Liem I, et al. Localizing the sentinel node in cutaneous melanoma: gamma probe detection versus blue dye. Ann Surg Oncol. 1997;4:156-60.

4. Chao C, Wong SL, Edwards MJ, et al. Sentinel lymph node biopsy for head and neck melanomas. Ann Surg Oncol. 2003;10: 21-6.

5. Eicher SA, Clayman GL, Myers JN, Gillenwater AM. A prospective study of intraoperative lymphatic mapping for head and neck cutaneous melanoma. Arch Otolaryngol Head Neck Surg. 2002;128:241-6.

6. Carlson GW, Page AJ, Cohen C, et al. Regional recurrence after negative sentinel lymph node biopsy for melanoma. Ann Surg. 2008;248:378-86.

7. Vermeeren L, van der Ploeg IMC, Valdés Olmos RA, Meinhardt W, Klop WMC, Kroon BBR, Nieweg OE. SPECT/CT for preoperative sentinel node localization. J Surg Oncol. 2010;101: 184-90.

8. Vermeeren L, Valdés Olmos RA, Klop WMC, van der Ploeg IMC, Nieweg OE, Balm AJM, van den Brekel MWM. SPECT/ CT for sentinel lymph node mapping in head and neck melanoma. Head Neck. 2010;33:1-6.

9. Vermeeren L, Klop WMC, van den Brekel MWM, Balm AJM, Nieweg OE, Valdés Olmos RA. Sentinel node detection in head and neck malignancies: innovations in radioguided surgery. $J$ Oncol. 2009;2009:681-746.

10. Vermeeren L, Valdés Olmos RA, Klop WMC, Balm AJM, van den Brekel MWM. A portable gamma-camera for intraoperative detection of sentinel nodes in the head and neck region. $J$ Nucl Med. 2010;51:700-3.

11. Govaert GAM, Oostenbroek RJ, Plaisier PW. Prolonged skin staining after intradermal use of patent blue in sentinel lymph node biopsy for breast cancer. Eur J Surg Oncol. 2005;31:373-5.

12. Vahrmeijer AL, Frangioni JV. Seeing the invisible during surgery. Br J Surg. 2011;98:749-50. 
13. Mieog JSD, Troyan SL, Hutteman M, et al. Toward optimization of imaging system and lymphatic tracer for near-infrared fluorescent sentinel lymph node mapping in breast cancer. Ann Surg Oncol. 2011;18:2483-91.

14. Hutteman M, Mieog JSD, van der Vorst JR, et al. Randomized, double-blind comparison of indocyanine green with or without albumin premixing for near-infrared fluorescence imaging of sentinel lymph nodes in breast cancer patients. Breast Cancer Res Treat. 2011;127:163-70.

15. Bredell MG. Sentinel lymph node mapping by indocyanine green fluorescence imaging in oropharyngeal cancer: preliminary experience. Head Neck Oncol. 2010;2:31.

16. Hayashi T, Furukawa H, Oyama A, Funayama E, Saito A, Yamao T, Yamamoto Y. Sentinel lymph node biopsy using real-time fluorescence navigation with indocyanine green in cutaneous head and neck/lip mucosa melanomas. Head Neck. 2011. doi: 10.1002/hed.21651.

17. van Leeuwen AC, Buckle $T$, Bendle G, Vermeeren L, Valdés Olmos RA, van de Poel HG, van Leeuwen FWB. Tracer-cocktail injections for combined pre- and intraoperative multimodal imaging of lymph nodes in a spontaneous mouse prostate tumor model. J Biomed Opt. 2011;16:016004.
18. Buckle T, van Leeuwen AC, Chin PTK, Janssen H, Muller SH, Jonkers J, van Leeuwen FWB. A self-assembled multimodal complex for combined pre- and intraoperative imaging of the sentinel lymph node. Nanotechnology. 2010;21:355101.

19. van der Poel HG, Buckle T, Brouwer OR, Valdés Olmos RA, van Leeuwen FWB. Intraoperative laparoscopic fluorescence guidance to the sentinel lymph node in prostate cancer patients: clinical proof of concept of an integrated functional imaging approach using a multimodal tracer. Eur Urol. 2011;60:826-33.

20. Nieweg OE, Tanis PJ, Kroon BB. The definition of a sentinel node. Ann Surg Oncol. 2001;8:538-41.

21. Keereweer S, Kerrebijn JDF, van Driel PBAA, et al. Optical image-guided surgery-where do we stand? Mol Imaging Biol. 2011;13:199-207.

22. Klop WMC, Veenstra HJ, Vermeeren L, Nieweg OE, Balm AJM, Lohuis PJFM. Assessment of lymphatic drainage patterns and implications for the extent of neck dissection in head and neck melanoma patients. J Surg Oncol. 2011;103:756-60.

23. Chin PTK, Buckle T, de Miguel AA, Meskers SCJ, Janssen RAJ, van Leeuwen FWB. Dual-emissive quantum dots for multispectral intraoperative fluorescence imaging. Biomaterials. 2010;31: 6823-32. 